Recepción: 20 / 04 / 2017

Aceptación: 20 / 05 / 2017

Publicación: 15 / 06 / 2017
Ciencias de la Educación

Artículo de Investigación

\title{
Guía relacionada al correcto manejo y aplicación de las TICs para Incrementar el desempeño escolar
}

\author{
Guide related to the correct handling and application of the TICs to Increase the \\ school performance
}

\section{Orientação relacionada com manuseio e aplicação das TIC para aumentar o desempenho escolar}

\author{
Eduardo G. Guadalupe-Coronel ${ }^{\mathrm{I}}$ \\ eduardo.guadalupeco@ug.edu.ec \\ Jonathan H. Guadalupe-Beltrán II \\ hans-jonathan@hotmail.com \\ Eduardo S. Guadalupe-Beltrán ${ }^{\text {III }}$ \\ ab_eguadalupe@outlook.es
}

Correspondencia: eduardo.guadalupeco@ug.edu.ec

I. Diploma Superior en Diseño Curricular por Competencias, Magister en Diseño Curricular, Abogado de los Tribunales y Juzgados de la República del Ecuador, Licenciado en Ciencias de la Educación Mención Informática, Profesor de Educación Media; Universidad de II. Guayaquil, Guayaquil, Ecuador.

Ingeniero Electrónico, Ecuador, Universidad Politécnica Salesiana, Guayaquil, Ecuador.

III. Ingeniero en Ciencias Empresariales; Abogado de los Tribunales y Juzgados de la República del Ecuador, Universidad Politécnica Salesiana, Guayaquil, Ecuador. 


\section{Resumen}

La tesis de maestría en Diseño curricular, se inicia con el problema pedagógico detectada en las aulas del octavo grado de educación general básica de la Unidad Educativa Fiscal Aguirre Abad de la ciudad de Guayaquil, lugar donde los aprendientes tienen enormes vacíos de contenidos tecnológicos que afectan el proceso de adquisición de conocimientos de primer nivel, además de la poca realización de tareas utilizando los medios idóneos. Como respuesta a ello, el investigador plantea una guía de aplicación de las Tecnologías de información y comunicación, junto a sus diferentes herramientas, las mismas que potenciarían la formación integral y por ende el rendimiento escolar y la motivación por el estudio. En la investigación, se detalló la metodología innovadora utilizada para el efecto, tanto con las modalidades como con los métodos y los niveles de profundidad, entre ellos la exploratoria, descriptiva y diagnóstica. Igualmente se determinó adecuadamente los objetivos del trabajo académico, así como la hipótesis o supuesto detallado por el maestrante. El marco teórico fue muy importante por la recolección de información sobre las Tics y sus estrategias para desarrollar actividades integradores con los estudiantes del referido paralelo. Se determinó con claridad la estadística descriptiva en la información relacionada a las encuestas y entrevistas efectuadas a las autoridades del plantel, Hodogogos, representantes legales y estudiantes, que apoyaron en forma abierta los detalles de cada pregunta señalada en el documento analizado. Los resultados recogidos determinaron la validez de la hipótesis y la adhesión a la propuesta de la guía motivacional diseñada por el autor de la tesis.

Palabras claves: Tecnología; internet; utilitarios. 


\section{Abstract}

The master's thesis in Curricular Design begins with the pedagogical problem detected in the eighth grade classrooms of basic education of the Fiscal Education Unit Aguirre Abad of the city of Guayaquil, where learners have huge gaps of technological contents that affect the process of acquiring first-level knowledge, in addition to the little accomplishment of tasks using the appropriate means. As a response to this, the researcher proposes a guide to the application of Information and Communication Technologies, along with their different tools, which would enhance comprehensive training and therefore school performance and motivation for study. In the investigation, the innovative methodology used for the effect was detailed, as well as the modalities as well as the methods and depth levels, including the exploratory, descriptive and diagnostic. The objectives of the academic work were also determined adequately, as well as the hypothesis or supposition detailed by the teacher. The theoretical framework was very important for the collection of information about the Tics and their strategies to develop integrative activities with the students of said parallel. The descriptive statistics in the information related to the surveys and interviews with the authorities of the site, Hodogogos, legal representatives and students, who openly supported the details of each question identified in the document analyzed, were clearly determined. The results obtained determined the validity of the hypothesis and the adherence to the proposal of the motivational guide designed by the author of the thesis.

Key words: Technology; Internet; Utilities. 


\section{Resumo}

Tese de mestrado em design de currículo, começa com o problema pedagógico detectado nas salas de aula oitava série do ensino fundamental da cidade Unidade de Educação Fiscal Aguirre Abad de Guayaquil, onde os alunos têm enormes lacunas conteúdo tecnológico afetando o processo de aquisição do primeiro conhecimento, para além das tarefas de desempenho de baixo através dos meios apropriados. Em resposta a isso, o pesquisador apresenta um guia para a aplicação de tecnologias de informação e comunicação, juntamente com suas várias ferramentas, a mesma que iria melhorar a educação integral e, assim, o desempenho do aluno e motivação para o estudo. Em investigação, a inovadora metodologia utilizada para o efeito, ambas as modalidades de como os métodos e os níveis de profundidade, incluindo exploratório, descritivo e de diagnóstico acima descrita. Da mesma forma os objetivos do trabalho acadêmico determinado corretamente, ea hipótese ou assunção pelo maestrante detalhado. O referencial teórico foi muito importante para a coleta de informações sobre as TIC e desenvolver estratégias para a integração de atividades com os alunos que se refere paralelo. Foi determinada estatísticas claramente descritivas sobre a informação relacionada com pesquisas e entrevistas com as autoridades escolares, Hodogogos, representantes legais e alunos, que apoiou abertamente os detalhes de cada pergunta indicada no documento analisado. Os resultados obtidos mostraram a validade da hipótese e adesão a proposta de guia motivacional projetado pelo autor da tese.

Palavras-chave: Tecnologia; Internet; Utilidade. 


\section{Introducción.}

Las instituciones educativas deben estar enmarcadas dentro de los estándares necesarios donde los docentes deben desarrollar en su quehacer diario el uso de competencias digitales que les permita acortar la brecha digital y generacional a través de estrategias metodológicas que permitan involucrar a los estudiantes al $100 \%$ y que sus aprendizajes sean aplicados para su vida diaria a través del uso de recursos tecnológicos como son las TIC. Estos recursos “están sirviendo de base para el surgimiento de un entorno completamente nuevo y diferente dentro del cual tendrán que desenvolverse los procesos de enseñanza y aprendizaje” (Brunner 2003:43)

La mayor parte de las instituciones poseen una gama de recursos tecnológicos como proyector, computadores, cámaras digitales que permiten potencializar los procesos de aprendizaje; "los principales factores que influyen en el uso de las TIC por parte de los docentes son: el acceso a este tipo de recursos, calidad de software y equipos, facilidad o simplicidad de uso, incentivo para cambiar las prácticas pedagógicas usando tecnología, el apoyo y solidaridad de las escuelas para usar las TIC en el currículo, las políticas nacionales y locales sobre TIC, compromiso con la superación profesional, capacitación formal recibida en el uso de las TIC.” (Mumtag, 2005)

En la Unidad Educativa fiscal Aguirre Abad de la ciudad de Guayaquil, es indispensable que los docentes se relacionen con el uso de las TIC's para lograr desarrollar nuevos aprendizajes significativos y por ende cambiar la actitud actual de los estudiantes del octavo grado. Cabe mencionar que las TIC “están sirviendo de base para el surgimiento de un entorno completamente nuevo y diferente dentro del cual tendrán que desenvolverse los procesos de enseñanza y aprendizaje" (Brunner 2003:43). 
El presente estudio tiene como objetivo aplicar una guía cognitiva sobre la correcta utilización de las TIC's, para optimizar el rendimiento escolar de los estudiantes de la Unidad Educativa Fiscal Aguirre Abad, con la finalidad de incrementar el desempeño docente y mejorar el proceso educativo. Una estrategia para lograr el objetivo es difundir en la comunidad educativa la importancia del uso de los instrumentos tecnológicos en la formación integral de los estudiantes; además se socializará un documento base sobre el uso básico de las herramientas TIC y de esta forma actualizar a los aprendientes del plantel en el uso de estas herramientas.

\section{Materiales y métodos.}

\section{Modalidades de la investigación}

El estudio se basa en dos modalidades de investigación:

Modalidad de Campo: Se basa en el estudio que permite la participación real del investigador o los investigadores, desde el mismo lugar donde ocurren los hechos. Refiriéndose a la modalidad de campo la tesis del autor se desarrolló en las aulas del octavo grado del colegio fiscal Aguirre Abad.

Modalidad de proyecto factible: Permite la solución de un problema de carácter práctico, que pueden conceder beneficios en diferentes áreas del acontecer diario. Cuando se determinó el problema de investigación se observó que el desarrollo de la tesis si era posible realizarlo, es decir, que el aporte de las autoridades, docentes, padres de familia y estudiantes fue decisivo para lograr la propuesta diseñada por el autor de la tesis.

\section{Métodos la investigación}

Los métodos utilizados para para llegar al objetivo de la tesis fueron: 
Métodos Empíricos: Utilizando la observación y la experiencia se pudo constar el problema del trabajo de la tesis al observar la poca y casi nula utilización de los insumos tecnológicos por parte de los aprendientes, lo cual incide en el rendimiento y coarta el desarrollo de los procesos educativos dentro del plantel

Método científico: Gracias al método científico, un investigador logra apartar su subjetividad y obtiene resultados más cercanos a la objetividad o a lo empírico

\section{Método hipotético- deductivo}

El método hipotético- deductivo es un método científico que considera que la conclusión se halla implícita dentro las premisas. Aplicando este método nos ha permitido partir de datos generales para llegar a casos muy específicos o particulares.

Método inductivo: Se trata del método científico más usual, en el que pueden distinguirse cuatro pasos esenciales: la observación de los hechos para su registro; la clasificación y el estudio de estos hechos; la derivación inductiva que parte de los hechos y permite llegar a una generalización; y la contrastación.

Método analítico: Este método nos permite conocer más del objeto de estudio, con lo cual se puede: explicar, hacer analogías, comprender mejor su comportamiento y establecer nuevas teorías.

Todos estos métodos por su importancia en la maestría de Diseño Curricular se los utilizo en los diferentes capítulos de la estructura de la tesis resaltando su importancia para el proceso de la investigación. 


\section{Tipo de investigación}

Nivel exploratorio: En este nivel realizamos una indagación previa o un sondeo para acercarnos al posible problema dentro del área de estudio el cual es el casi nulo desarrollo de competencias digitales de los docentes en el desarrollo de sus clases y en su ámbito personal

Nivel diagnóstico: Consiste en averiguar e identificar el problema existe en la institución educativa, analizando los antecedentes que lo originan para tener mayor certeza de lo que se investiga y dar alternativas de soluciones entre los involucrados y se elimine el problema pedagógico presentado en el aula.

\section{Población y muestra}

Población: La población está constituida por: Autoridades, Docentes, Representantes legales,

Estudiantes

\section{CUADRO DE LA POBLACIÓN}

\begin{tabular}{|l|l|l|}
\hline INVOLUCRADOS & No. & $\%$ \\
\hline AUTORIDADES & 2 & 2.94 \\
\hline DOCENTES & 8 & 8.82 \\
\hline PADRES DE FAMILIA & 43 & 44.12 \\
\hline ESTUDIANTES & 43 & 44.12 \\
\hline TOTAL & 96 & $100 \%$ \\
\hline
\end{tabular}

Fuente Secretaría del Plantel

\section{MUESTRA}

CUADRO DE LA MUESTRA

\begin{tabular}{|l|l|l|l|}
\hline INVOLUCRADOS & POBLACIÓN & MUESTRA & $\%$ \\
\hline AUTORIDADES & 2 & 3 & 3.26 \\
\hline DOCENTES & 8 & 8 & 9.78 \\
\hline PADRES DE FAMILIA & 43 & 40 & 43.48 \\
\hline ESTUDIANTES & 43 & 40 & 43.48 \\
\hline TOTAL & 96 & 91 & 100.00 \\
\hline
\end{tabular}


Eduardo G. Guadalupe-Coronel; Jonathan H. Guadalupe-Beltrán; Eduardo S. Guadalupe-Beltrán

\section{TÉCNICAS E INSTRUMENTO DE LA INVESTIGACIÓN}

El mecanismo que permitió recolectar los datos de los hechos investigados fue la encuesta. Es instrumento buscó recaudar datos por medio de un cuestionario prediseñado. Los datos se obtuvieron a partir de realizar un conjunto de preguntas normalizadas dirigidas a la muestra representativa.

\section{LEVANTAMIENTO DE DATOS}

La información se recolecto mediante la encuesta diseñada por el autor de la investigación, formulando las preguntas de carácter científico en mérito al tema y al problema pedagógico. La encuesta fue aplicada a las autoridades, docentes, representantes legales, estudiantes de la institución. Las preguntas cerradas fueron elaboradas en hojas individuales de acuerdo a la escala de Likert y se la realizó una sola vez el 14 de octubre del 2013 en las propias instalaciones de la unidad educativa fiscal Aguirre Abad. 


\section{Resultados.}

A continuación, se presenta la pregunta realizada en la encuesta, seguida de los datos estadísticos procesados y el respectivo análisis.

Pregunta No .1 ¿Los docentes de la institución están desactualizados en el ámbito de las innovaciones tecnológicas?

CUADRO NO. 1 AUTORIDAD EDUCATIVA

\begin{tabular}{|c|c|c|c|c|}
\hline$\overline{\text { ESCALA }}$ & ALTERNATIVAS & FRECUENCIA & $\%$ & \multirow{7}{*}{$\begin{array}{l}\text { El } 100 \% \text { de los encuestados respondió } \\
\text { muy de acuerdo con la pregunta } \\
\text { formulada en base a sus experiencias } \\
\text { profesionales. Esto indica que los } \\
\text { docentes de la institución están } \\
\text { desactualizados en el ámbito de la } \\
\text { tecnología educativa y por ende puede } \\
\text { causar incidencia dentro del proceso de } \\
\text { aprendizaje de los estudiantes, en las } \\
\text { diferentes asignaturas. }\end{array}$} \\
\hline 5 & MUY DE ACUERDO & 2 & $100 \%$ & \\
\hline 4 & DE ACUERDO & $\overline{0}$ & $\overline{0}$ & \\
\hline 3 & INDIFERENTE & 0 & 0.00 & \\
\hline 2 & EN DESACUERDO & 0 & 0.00 & \\
\hline 1 & $\begin{array}{l}\text { MUY EN } \\
\text { DESACUERDO }\end{array}$ & 0 & 0.00 & \\
\hline TOTAL & & 2 & 100.00 & \\
\hline
\end{tabular}

Pregunta No. 2 ¿Deben los docentes, capacitarse en forma óptima en conocimientos sobre tecnologías para el aprendizaje?

CUADRO NO. 2 AUTORIDAD EDUCATIVA

\begin{tabular}{|c|c|c|c|c|}
\hline ESCALA & ALTERNATIVAS & $\begin{array}{c}\text { FRECUENC } \\
\text { IA }\end{array}$ & $\%$ & \multirow{7}{*}{$\begin{array}{l}\text { Las autoridades del plantel } \\
\text { respondieron en un } 100 \% \text { estar muy } \\
\text { de acuerdo con que los docentes } \\
\text { actualicen sus conocimientos en el } \\
\text { marco de las tecnologías y sus } \\
\text { diferentes plataformas vinculadas a } \\
\text { los aprendizajes significativos, por } \\
\text { cuanto es de carácter beneficioso } \\
\text { para la institución al contar con un } \\
\text { personal capacitado acorde a la } \\
\text { realidad educativa actual y favorecer } \\
\text { la formación integral de los } \\
\text { estudiantes. }\end{array}$} \\
\hline 5 & $\begin{array}{c}\text { MUY DE } \\
\text { ACUERDO }\end{array}$ & 2 & 100.00 & \\
\hline 4 & DE ACUERDO & 0 & 0.00 & \\
\hline 3 & INDIFERENTE & 0 & 0.00 & \\
\hline 2 & EN DESACUERDO & 0 & 0.00 & \\
\hline 1 & $\begin{array}{c}\text { MUY EN } \\
\text { DESACUERDO }\end{array}$ & 0 & 0.00 & \\
\hline & TOTAL & 2 & 100.00 & \\
\hline
\end{tabular}

Pregunta No. 3 ¿Mejorarían los procesos de enseñanza de los docentes, utilizando apropiadamente las tecnologías educativas?

CUADRO NO. 3 AUTORIDAD EDUCATIVA

\begin{tabular}{|c|c|c|c|c|}
\hline ESCALA & ALTERNATIVAS & $\begin{array}{l}\text { FRECUENC } \\
\text { IA }\end{array}$ & $\%$ & \multirow{7}{*}{$\begin{array}{l}\text { El } 50 \% \text { de las autoridades del } \\
\text { plantel está muy de acuerdo, } \\
\text { mientras tanto el otro } 50 \% \text { están } \\
\text { de acuerdo en que sería altamente } \\
\text { provechoso para la institución } \\
\text { educativa que los Hodogogos del } \\
\text { plantel, obtengan una capacitación } \\
\text { excelente en lo relacionada al uso } \\
\text { de las tecnologías }\end{array}$} \\
\hline 5 & MUY DE ACUERDO & 1 & $50 \%$ & \\
\hline 4 & DE ACUERDO & 1 & $50 \%$ & \\
\hline 3 & INDIFERENTE & 0 & 0.00 & \\
\hline 2 & EN DESACUERDO & 0 & 0.00 & \\
\hline 1 & $\begin{array}{l}\text { MUY EN } \\
\text { DESACUERDO }\end{array}$ & 0 & 0.00 & \\
\hline$\overline{\text { TOT }}$ & & 2 & 100.00 & \\
\hline
\end{tabular}


Pregunta No. 4 ¿Es apropiado difundir una guía cognitiva sobre la correcta aplicación de las Tics en el aprendizaje de los estudiantes del octavo grado del plantel?

CUADRO NO. 4 AUTORIDAD EDUCATIVA

\begin{tabular}{|c|c|c|c|c|}
\hline ESCALA & ALTERNATIVAS & $\begin{array}{c}\text { FRECUEN } \\
\text { CIA }\end{array}$ & $\%$ & \multirow{7}{*}{$\begin{array}{l}\text { El } 100 \% \text { de las autoridades opinan } \\
\text { que están muy de acuerdo que se } \\
\text { debe aplicar una guía cognitiva } \\
\text { relacionada al conocimiento y } \\
\text { aplicación de las Tics, con la } \\
\text { finalidad de potenciar los } \\
\text { aprendizajes y habilidades de los } \\
\text { estudiantes del octavo grado del } \\
\text { colegio. }\end{array}$} \\
\hline 5 & MUY DE ACUERDO & 2 & 100.00 & \\
\hline 4 & DE ACUERDO & 0 & 0.00 & \\
\hline 3 & INDIFERENTE & 0 & 0.00 & \\
\hline 2 & EN DESACUERDO & 0 & 0.00 & \\
\hline 1 & $\begin{array}{c}\text { MUY EN } \\
\text { DESACUERDO }\end{array}$ & 0 & 0.00 & \\
\hline & TOTAL & 2 & 100.00 & \\
\hline
\end{tabular}

Pregunta No. 5 ¿Considera necesario que los contenidos sobre las Tics se impartan obligatoriamente por los docentes de las diferentes asignaturas?

CUADRO NO. 5 AUTORIDAD EDUCATIVA

\begin{tabular}{|c|c|c|c|c|}
\hline ESCALA & ALTERNATIVAS & $\begin{array}{l}\text { FRECUEN } \\
\text { CIA }\end{array}$ & $\%$ & \multirow{7}{*}{$\begin{array}{l}\text { El } 100 \% \text { de las autoridades del } \\
\text { plantel están muy de acuerdo con la } \\
\text { pregunta formulada por el maestrante, } \\
\text { es decir que los docentes del plantel, } \\
\text { deben impartir sus clases diarias, } \\
\text { apoyándose e n la tecnología } \\
\text { educativa y así mejorar el } \\
\text { rendimiento escolar de los } \\
\text { estudiantes. }\end{array}$} \\
\hline 5 & MUY DE ACUERDO & 2 & 100.00 & \\
\hline 4 & DE ACUERDO & 0 & 0.00 & \\
\hline 3 & INDIFERENTE & 0 & 0.00 & \\
\hline 2 & EN DESACUERDO & 0 & 0.00 & \\
\hline 1 & $\begin{array}{l}\text { MUY EN } \\
\text { DESACUERDO }\end{array}$ & 0 & 0.00 & \\
\hline TOTAL & & 2 & 100.00 & \\
\hline
\end{tabular}

Pregunta No. 6 ¿Los docentes deben utilizar herramientas relacionadas a las Tics en cada una de sus clases?

CUADRO NO. 6 AUTORIDAD EDUCATIVA

\begin{tabular}{|c|c|c|c|c|}
\hline$\overline{\text { ESCALA }}$ & ALTERNATIVAS & $\begin{array}{l}\text { FRECUEN } \\
\text { CIA }\end{array}$ & $\%$ & \multirow{7}{*}{$\begin{array}{l}\text { Las autoridades del plantel en un } \\
100 \% \text { están muy de acuerdo en que los } \\
\text { docentes deben utilizar herramientas } \\
\text { vinculadas a las Tics en cada de sus } \\
\text { clases por cuanto esto permitirá que } \\
\text { puedan manejar herramientas y } \\
\text { programas que le permitan ampliar sus } \\
\text { estrategias metodológicas y recursos } \\
\text { didácticos, para alcanzar logros } \\
\text { significativos con sus estudiantes. }\end{array}$} \\
\hline 5 & MUY DE ACUERDO & 2 & 100.00 & \\
\hline 4 & DE ACUERDO & 0 & 0.00 & \\
\hline 3 & INDIFERENTE & 0 & 0.00 & \\
\hline 2 & EN DESACUERDO & 0 & 0.00 & \\
\hline 1 & $\begin{array}{l}\text { MUY EN } \\
\text { DESACUERDO }\end{array}$ & 0 & 0.00 & \\
\hline TOTAL & & 2 & 100.00 & \\
\hline
\end{tabular}


Pregunta No. 7 ¿Sería oportuno que los docentes del plantel asistan a cursos sobre tecnologías educativas y además capacitarse para aplicar esos conocimientos y habilidades en sus clases?

CUADRO NO. 7 AUTORIDAD EDUCATIVA

\begin{tabular}{|c|c|c|c|c|}
\hline ESCALA & ALTERNATIVAS & $\begin{array}{l}\text { FRECUENC } \\
\text { IA }\end{array}$ & $\%$ & \multirow{7}{*}{$\begin{array}{l}\text { El 50\% de las autoridades } \\
\text { consideran estar muy de acuerdo } \\
\text { mientras que el restante } 50 \% \\
\text { contestaron que están de acuerdo } \\
\text { que los docentes del plantel asistan } \\
\text { a cursos relacionadas al } \\
\text { conocimiento y uso de las } \\
\text { tecnologías educativas }\end{array}$} \\
\hline 5 & MUY DE ACUERDO & 1 & $50 \%$ & \\
\hline 4 & DE ACUERDO & 1 & $50 \%$ & \\
\hline 3 & INDIFERENTE & 0 & 0.00 & \\
\hline 2 & EN DESACUERDO & 0 & 0.00 & \\
\hline 1 & $\begin{array}{l}\text { MUY EN } \\
\text { DESACUERDO }\end{array}$ & 0 & 0.00 & \\
\hline TOTAL & & 2 & 100.00 & \\
\hline
\end{tabular}

Pregunta No. 8 ¿El uso de herramientas como Word, Excel y PowerPoint deben ser parte de los contenidos que el docente debe manejar dentro de su planificación en clase de su asignatura?

CUADRO NO. 8 AUTORIDAD EDUCATIVA

\begin{tabular}{|c|c|c|c|c|}
\hline ESCALA & ALTERNATIVAS & $\begin{array}{l}\text { FRECUEN } \\
\text { CIA }\end{array}$ & $\%$ & \multirow{7}{*}{$\begin{array}{l}\text { Las autoridades del plantel están muy } \\
\text { de acuerdo en un } 100 \% \text { en referencia a } \\
\text { que los docentes deben manejar } \\
\text { correctamente los programas de Office } \\
\text { además de Internet por cuanto deben ser } \\
\text { herramientas en su trabajo diario que } \\
\text { deben usar e incluirlos en su } \\
\text { planificación para mejorar el } \\
\text { aprendizaje de los estudiantes, al igual } \\
\text { que su rendimiento escolar. }\end{array}$} \\
\hline 5 & $\begin{array}{l}\text { MUY DE } \\
\text { ACUERDO }\end{array}$ & 2 & $\begin{array}{l}100.0 \\
0\end{array}$ & \\
\hline 4 & DE ACUERDO & 0 & 0.00 & \\
\hline 3 & INDIFERENTE & 0 & 0.00 & \\
\hline 2 & EN DESACUERDO & 0 & 0.00 & \\
\hline 1 & $\begin{array}{l}\text { MUY EN } \\
\text { DESACUERDO }\end{array}$ & 0 & 0.00 & \\
\hline TOTAL & & 2 & 100.0 & \\
\hline
\end{tabular}

Pregunta No. 1 ¿Utiliza las Tecnologías Educativas en su profesión educativa y personal?

CUADRO NO. 9 DOCENTES

\begin{tabular}{|c|c|c|c|c|}
\hline ESCALA & ALTERNATIVAS & $\begin{array}{c}\text { FRECUENC } \\
\text { IA }\end{array}$ & $\%$ & $\begin{array}{l}\text { Los docentes en un 22.22\% están muy } \\
\text { de acuerdo; el 11.11\% está de acuerdo; } \\
\text { el 11.11\% respondió estar indiferente; } \\
\text { el } 33.33 \% \text { están en desacuerdo } \\
\text { mientras que el 22.22\% respondió estar } \\
\text { muy en desacuerdo; existió una } \\
\text { diversidad de respuestas pero el } \\
\text { análisis final es que los docentes no se } \\
\text { involucran con las tecnologías } \\
\text { educativas en lo personal y en lo } \\
\text { educativo, por lo que se debe impulsar } \\
\text { estas acciones en beneficio de la } \\
\text { institución educativa. }\end{array}$ \\
\hline 3 & DE ACUERDO & 1 & 11.11 & 11.11 \\
\hline 1 & INDIFERENTE & 1 & 33.33 & 22.22 \\
\hline
\end{tabular}


Pregunta No. 2 ¿Cree usted que es necesario estar actualizados en el ámbito de la Tecnología educativa, para beneficio de los aprendientes?

CUADRO N.- 10 DOCENTES

\begin{tabular}{|l|l|l|l|l|}
\hline ESCALA & ALTERNATIVAS & $\begin{array}{l}\text { FRECUENC } \\
\text { IA }\end{array}$ & $\%$ & $\begin{array}{l}\text { Los docentes encuestados contestaron } \\
\text { en un 66.67\% estar muy de acuerdo; el } \\
22.22 \% \text { aseguro estar de acuerdo y el } \\
11.1 \% \text { aseveraron estar indiferentes a } \\
\text { la necesidad de estar actualizados en el }\end{array}$ \\
\hline 5 & $\begin{array}{l}\text { MUY DE } \\
\text { ACUERDO }\end{array}$ & 6 & 66.67 & $\begin{array}{l}\text { ámbito de la Tecnología educativa, } \\
\text { ellos opinan que los docentes necesitan } \\
\text { estar capacitados y preparados ante los } \\
\text { nuevos avances tecnológicos para } \\
\text { aplicarlos en el desarrollo de las } \\
\text { asignaturas del currículo. }\end{array}$ \\
\hline 3 & DE ACUERDO & 2 & 33.33 \\
\hline 1 & INDIFERENTE & 0 & 0 & 0.00 \\
\hline
\end{tabular}

Pregunta No. 3 ¿Los estudiantes se motivan cuando usted utiliza las herramientas tecnológicas en el aula?

CUADRO N.- 11 DOCENTES

\begin{tabular}{|c|c|c|c|c|}
\hline$\overline{\text { ESCALA }}$ & ALTERNATIVAS & $\begin{array}{l}\text { FRECUENC } \\
\text { IA }\end{array}$ & $\%$ & \multirow{7}{*}{$\begin{array}{l}\text { Los docentes en un 55.56\% } \\
\text { respondieron estar muy de acuerdo; } \\
\text { el } 33.33 \% \text { opino estar de acuerdo y } \\
\text { el } 11.11 \% \text { indicaron ser indiferente } \\
\text { cuando el docente utiliza las } \\
\text { tecnologías educativas en las clases } \\
\text { dejado atrás recursos tradicionales e } \\
\text { incorporando nuevos que logren la } \\
\text { mayor atención del estudiante en el } \\
\text { aula y así mejorar la formación } \\
\text { integral de los aprendientes. }\end{array}$} \\
\hline 5 & $\begin{array}{l}\text { MUY DE } \\
\text { ACUERDO }\end{array}$ & 4 & 55.56 & \\
\hline 4 & DE ACUERDO & 3 & 33.33 & \\
\hline 3 & INDIFERENTE & 1 & 11.11 & \\
\hline 2 & $\begin{array}{l}\text { EN } \\
\text { DESACUERDO }\end{array}$ & 0 & 0.00 & \\
\hline 1 & $\begin{array}{l}\text { MUY EN } \\
\text { DESACUERDO }\end{array}$ & 0 & 0.00 & \\
\hline TOTAL & & 8 & 100.00 & \\
\hline
\end{tabular}

Pregunta No. 4 ¿Aceptaría colaborar en la difusión de una guía de sobre el uso educativo de las Tics, en beneficio de los estudiantes del octavo grado?

CUADRO N.- 12 DOCENTES

\begin{tabular}{|c|c|c|c|c|}
\hline $\begin{array}{l}\text { ESCAL } \\
\text { A } \\
\end{array}$ & ALTERNATIVAS & $\begin{array}{l}\text { FRECUENC } \\
\text { IA }\end{array}$ & $\%$ & \multirow{7}{*}{$\begin{array}{l}\text { En un } 66.67 \% \text { los docentes están muy de } \\
\text { acuerdo; un } 22.22 \% \text { está de acuerdo } \\
\text { mientras que el } 11.11 \% \text { es indiferente a } \\
\text { la pregunta: la alternativa más alta es } \\
\text { que los docentes están muy de acuerdo } \\
\text { en difundir una guía sobre el correcto } \\
\text { uso de las Tics que les serviría a los } \\
\text { estudiantes para identificar las } \\
\text { herramientas y programas que deben } \\
\text { revisar y manejar. }\end{array}$} \\
\hline 5 & $\begin{array}{l}\text { MUY DE } \\
\text { ACUERDO }\end{array}$ & 5 & 66.67 & \\
\hline 4 & DE ACUERDO & 2 & 22.22 & \\
\hline 3 & INDIFERENTE & 1 & 11.11 & \\
\hline 2 & EN DESACUERDO & 0 & 0.00 & \\
\hline 1 & $\begin{array}{l}\text { MUY EN } \\
\text { DESACUERDO }\end{array}$ & 0 & 0.00 & \\
\hline TOTAL & & 8 & 100.00 & \\
\hline
\end{tabular}


Pregunta No. 5 ¿El conocimiento de las tecnologías de información y comunicación, fortalecen a los docentes como base de su mejoramiento profesional?

\begin{tabular}{|c|c|c|c|c|}
\hline \multicolumn{5}{|c|}{ CUADRO N.- 13 DOCENTES } \\
\hline ESCALA & ALTERNATIVAS & $\begin{array}{l}\text { FRECUENC } \\
\text { IA }\end{array}$ & $\%$ & \multirow{7}{*}{$\begin{array}{l}\text { Los docentes en un } 44.44 \% \text { están muy de } \\
\text { acuerdo; el } 22.22 \% \text { está de acuerdo; el } \\
11.11 \% \text { respondió estar indiferente } \\
\text { mientras que el } 22.22 \% \text { respondió estar } \\
\text { en desacuerdo; existió una diversidad de } \\
\text { respuestas pero el análisis final es que los } \\
\text { docentes consideran que se deben } \\
\text { preparar eficientemente con la finalidad } \\
\text { de seguir preparándose para su } \\
\text { mejoramiento profesional. }\end{array}$} \\
\hline 5 & $\begin{array}{l}\text { MUY DE } \\
\text { ACUERDO }\end{array}$ & - & 44.44 & \\
\hline 4 & DE ACUERDO & 2 & 22.22 & \\
\hline 3 & INDIFERENTE & 1 & 11.11 & \\
\hline 2 & EN DESACUERDO & 2 & 22.22 & \\
\hline 1 & $\begin{array}{l}\text { MUY EN } \\
\text { DESACUERDO }\end{array}$ & 0 & 0.00 & \\
\hline TOTAL & & 8 & 100.00 & \\
\hline
\end{tabular}

Pregunta No. 6 ¿los docentes deberían utilizar herramientas TIC en sus clases diarias con sus estudiantes?

CUADRO N.- 14 DOCENTES

\begin{tabular}{|c|c|c|c|c|}
\hline ESCALA & ALTERNATIVAS & $\begin{array}{l}\text { FRECUENC } \\
\text { IA }\end{array}$ & $\%$ & \multirow{7}{*}{$\begin{array}{l}\text { El } 55.56 \% \text { respondió estar muy de } \\
\text { acuerdo; el } 22.22 \% \text { de acuerdo; el } 11.11 \% \\
\text { indiferente mientras tanto que el } 11.11 \% \\
\text { respondió estar en desacuerdo analizando } \\
\text { las respuestas, es evidente que los } \\
\text { docentes consideran que deben utilizar las } \\
\text { herramientas TIC en sus clases como un } \\
\text { recurso y estrategia para conseguir el } \\
\text { aprendizaje significativo en los } \\
\text { aprendientes del plantel. }\end{array}$} \\
\hline 5 & $\begin{array}{l}\text { MUY DE } \\
\text { ACUERDO }\end{array}$ & 4 & 55.56 & \\
\hline 4 & DE ACUERDO & 2 & 22.22 & \\
\hline 3 & INDIFERENTE & 1 & 11.11 & \\
\hline 2 & EN DESACUERDO & 1 & 11.11 & \\
\hline 1 & $\begin{array}{l}\text { MUY EN } \\
\text { DESACUERDO }\end{array}$ & 0 & 0.00 & \\
\hline TOTAL & & $\overline{8}$ & 100.00 & \\
\hline
\end{tabular}

Pregunta No. 7 ¿Los estudiantes exigen a los docentes planificar sus clases, basados en la tecnología educativa?

CUADRO N.- 15 DOCENTES

\begin{tabular}{|c|c|c|c|c|}
\hline ESCALA & ALTERNATIVAS & $\begin{array}{l}\text { FRECUENC } \\
\text { IA }\end{array}$ & $\%$ & \multirow{7}{*}{$\begin{array}{l}\text { En un } 44.44 \% \text { los docentes respondieron } \\
\text { estar muy de acuerdo; el } 22.22 \% \\
\text { respondió estar de acuerdo; el otro } \\
22.22 \% \text { considero estar indiferente y el } \\
11.11 \% \text { en desacuerdo en referencia a que } \\
\text { los aprendientes exigen que los docentes } \\
\text { deben aplicar las Tics en cada una de las } \\
\text { clases de las diferentes asignaturas de la } \\
\text { malla curricular. }\end{array}$} \\
\hline 5 & $\begin{array}{l}\text { MUY DE } \\
\text { ACUERDO }\end{array}$ & 3 & 44.44 & \\
\hline 4 & DE ACUERDO & 2 & 22.22 & \\
\hline 3 & INDIFERENTE & 2 & 22.22 & \\
\hline 2 & EN DESACUERDO & 1 & 11.11 & \\
\hline 1 & $\begin{array}{l}\text { MUY EN } \\
\text { DESACUERDO }\end{array}$ & 0 & 0.00 & \\
\hline TOTAL & & 8 & 100.00 & \\
\hline
\end{tabular}


Pregunta No. 8 ¿Conocen los docentes los utilitarios Word, Excel y PowerPoint, además del uso de internet?

\begin{tabular}{|c|c|c|c|c|}
\hline \multicolumn{5}{|c|}{ CUADRO N.- 16 DOCENTES } \\
\hline $\begin{array}{l}\text { ESCAL } \\
\text { A }\end{array}$ & ALTERNATIVAS & $\begin{array}{l}\text { FRECUENC } \\
\text { IA }\end{array}$ & $\%$ & \multirow{7}{*}{$\begin{array}{l}\text { Los docentes en un 55.56\% respondieron } \\
\text { estar muy de acuerdo; el } 33.33 \% \\
\text { considero estar de acuerdo y el } 11.11 \% \\
\text { restante respondió indiferente en esta } \\
\text { pregunta, por lo que los docentes } \\
\text { consideran que es necesario el uso de } \\
\text { programas utilitarios Word, Excel y } \\
\text { PowerPoint, además del uso de internet } \\
\text { para aplicarlos en las asignaturas de tanto } \\
\text { de octavo grado como del resto de los } \\
\text { paralelo de educación básica y del } \\
\text { bachillerato en general. }\end{array}$} \\
\hline 5 & $\begin{array}{l}\text { MUY DE } \\
\text { ACUERDO }\end{array}$ & 4 & 55.56 & \\
\hline 4 & DE ACUERDO & 3 & 33.33 & \\
\hline 3 & INDIFERENTE & 1 & 11.11 & \\
\hline 2 & EN DESACUERDO & 0 & 0.00 & \\
\hline 1 & $\begin{array}{l}\text { MUY EN } \\
\text { DESACUERDO }\end{array}$ & 0 & 0.00 & \\
\hline \multicolumn{2}{|l|}{ TOTAL } & 8 & $\mathbf{1 0 0 . 0 0}$ & \\
\hline
\end{tabular}

Pregunta No. 1 ¿Los docentes de octavo grado, tienen conocimientos básicos de las diferentes herramientas tecnológicas?

CUADRO No. 17 REPRESENTANTES LEGALES

\begin{tabular}{|c|c|c|c|c|}
\hline$\overline{\text { ESCALA }}$ & ALTERNATIVAS & $\begin{array}{l}\text { FRECUENCI } \\
\text { A }\end{array}$ & $\%$ & \multirow{7}{*}{$\begin{array}{l}\text { Los padres de familia en un } 42.50 \% \text { están } \\
\text { muy de acuerdo; el } 25 \% \text { de acuerdo; el } \\
5 \% \text { le pareció indiferente; el } 20 \% \text { en } \\
\text { desacuerdo y el } 7.50 \% \text { muy en } \\
\text { desacuerdo. Las respuestas conducen a } \\
\text { reflexionar el descuido de varios } \\
\text { profesores en prepararse adecuadamente } \\
\text { en el conocimiento de las Tics y sus } \\
\text { utilitarios para aplicarlos en el desarrollo } \\
\text { de los temas de las asignaturas de octavo } \\
\text { grado. }\end{array}$} \\
\hline 5 & $\begin{array}{l}\text { MUY DE } \\
\text { ACUERDO }\end{array}$ & 17 & 42.50 & \\
\hline 4 & DE ACUERDO & 10 & 25.00 & \\
\hline 3 & INDIFERENTE & 2 & 5.00 & \\
\hline 2 & $\begin{array}{l}\text { EN } \\
\text { DESACUERDO }\end{array}$ & 8 & 20.00 & \\
\hline 1 & $\begin{array}{l}\text { MUY EN } \\
\text { DESACUERDO }\end{array}$ & 3 & 7.50 & \\
\hline TOTAL & & 40 & 100.00 & \\
\hline
\end{tabular}

Pregunta No. 2 ¿Los docentes conocen el correcto uso de la tecnología para potenciar la educación de sus hijos?

CUADRO No. 18 REPRESENTANTES LEGALES

\begin{tabular}{|c|c|c|c|c|}
\hline $\begin{array}{l}\text { ESCA } \\
\text { LA }\end{array}$ & ALTERNATIVAS & $\begin{array}{l}\text { FRECUENCI } \\
\text { A }\end{array}$ & $\%$ & \multirow{7}{*}{$\begin{array}{l}\text { En un } 75 \% \text { los padres de familia } \\
\text { consideran estar muy de acuerdo; en } \\
\text { un } 15 \% \text { están de acuerdo mientras } \\
\text { que en un } 10 \% \text { responden indiferente. } \\
\text { Es evidente que los padres de familia } \\
\text { están conscientes de que los docentes } \\
\text { deben estar al día con los avances } \\
\text { tecnológicos en el campo educacional } \\
\text { por cuanto esto beneficiaría a la } \\
\text { comunidad educativa y al prestigio } \\
\text { del colegio fiscal Aguire Abad. }\end{array}$} \\
\hline 5 & $\begin{array}{l}\text { MUY DE } \\
\text { ACUERDO }\end{array}$ & 30 & 75.00 & \\
\hline 4 & DE ACUERDO & 6 & 15.00 & \\
\hline 3 & INDIFERENTE & 4 & 10.00 & \\
\hline 2 & EN DESACUERDO & 0 & 0.00 & \\
\hline 1 & $\begin{array}{l}\text { MUY EN } \\
\text { DESACUERDO }\end{array}$ & 0 & 0.00 & \\
\hline \multicolumn{2}{|c|}{ TOTAL } & 40 & 100.00 & \\
\hline
\end{tabular}


Pregunta No. 3 ¿Colaboraría con los docentes de octavo grado para ampliar y crear un ambiente propicio para el aprendizaje de sus representados?

\section{CUADRO No. 19 REPRESENTANTES LEGALES}

\begin{tabular}{|c|c|c|c|c|}
\hline $\begin{array}{l}\text { ESCA } \\
\text { LA }\end{array}$ & ALTERNATIVAS & $\begin{array}{l}\text { FRECUENCI } \\
\text { A }\end{array}$ & $\%$ & \multirow{7}{*}{$\begin{array}{l}\text { Los padres de familia en un } 65 \% \\
\text { respondieron estar muy de acuerdo; el } \\
10 \% \text { de acuerdo; el } 5 \% \text { indiferente; el } \\
15 \% \text { respondió estar en desacuerdo } \\
\text { mientras que el restante } 5 \% \text { están muy en } \\
\text { desacuerdo. Los padres opinan que } \\
\text { siempre apoyarían las iniciativas de los } \\
\text { docentes sobre el uso correcto de las } \\
\text { tecnologías educativas, ya que las } \\
\text { mismas beneficiarían a sus hijos por } \\
\text { cuanto se sentirían en un ambiente más } \\
\text { propicio para estudiar. }\end{array}$} \\
\hline 5 & $\begin{array}{l}\text { MUY DE } \\
\text { ACUERDO }\end{array}$ & 26 & 65.00 & \\
\hline 4 & DE ACUERDO & 4 & 10.00 & \\
\hline 3 & INDIFERENTE & 2 & 5.00 & \\
\hline 2 & EN DESACUERDO & 6 & 15.00 & \\
\hline 1 & $\begin{array}{l}\text { MUY EN } \\
\text { DESACUERDO }\end{array}$ & 2 & 5.00 & \\
\hline \multicolumn{2}{|c|}{ TOTAL } & 40 & 100.00 & \\
\hline
\end{tabular}

Pregunta No. 4 ¿Compartiría la difusión de una guía sobre el uso correcto de las Tics y su aplicación en los estudiantes de octavo grado?

CUADRO No. 20 REPRESENTANTES LEGALES

\begin{tabular}{|c|c|c|c|c|}
\hline $\begin{array}{l}\text { ESCA } \\
\text { LA } \\
\end{array}$ & ALTERNATIVAS & $\begin{array}{l}\text { FRECUENCI } \\
\text { A }\end{array}$ & $\%$ & \multirow{7}{*}{$\begin{array}{l}\text { En un } 77.50 \% \text { respondieron estar muy } \\
\text { de acuerdo; un } 15 \% \text { considero estar de } \\
\text { acuerdo y el } 7.50 \% \text { indiferente. Los } \\
\text { padres de familia en un porcentaje alto } \\
\text { creen que es primordial la difusión de } \\
\text { una guía cognitiva sobre el correcto uso } \\
\text { de las tecnologías de información y } \\
\text { comunicación para el área educativa y } \\
\text { particularmente para beneficiar a sus } \\
\text { representados del octavo grado. }\end{array}$} \\
\hline 5 & $\begin{array}{l}\text { MUY DE } \\
\text { ACUERDO }\end{array}$ & 30 & 77.50 & \\
\hline 4 & DE ACUERDO & 7 & 17.50 & \\
\hline 3 & INDIFERENTE & 3 & 7.50 & \\
\hline 2 & EN DESACUERDO & 0 & 0.00 & \\
\hline 1 & $\begin{array}{l}\text { MUY EN } \\
\text { DESACUERDO }\end{array}$ & 0 & 0.00 & \\
\hline \multicolumn{2}{|c|}{ TOTAL } & 40 & 100.00 & \\
\hline
\end{tabular}

Pregunta No. 5 ¿Se preocupa usted del rendimiento escolar de su representado y lo ayuda en casa en sus tareas escolares?

CUADRO No. 21 REPRESENTANTES LEGALES

\begin{tabular}{|c|c|c|c|c|}
\hline $\begin{array}{l}\text { ESCA } \\
\text { LA }\end{array}$ & ALTERNATIVAS & ARECUENCI & $\%$ & \multirow{7}{*}{$\begin{array}{l}\text { Los padres de familia en un } 87.50 \% \\
\text { respondieron estar muy de acuerdo; el } \\
7.50 \text { está de acuerdo mientras que el } 5 \% \\
\text { están indiferente ante la pregunta. El } \\
\text { porcentaje evidencia que existe una } \\
\text { preocupación de los padres de familia en } \\
\text { observar muy de cerca el rendimiento } \\
\text { escolar de sus representados y que } \\
\text { siempre están prestos a ayudarlos en las } \\
\text { tareas en el hogar. }\end{array}$} \\
\hline 5 & $\begin{array}{l}\text { MUY DE } \\
\text { ACUERDO }\end{array}$ & 35 & 87.50 & \\
\hline 4 & DE ACUERDO & 3 & 7.50 & \\
\hline 3 & INDIFERENTE & 2 & 5.00 & \\
\hline 2 & EN DESACUERDO & 0 & 0.00 & \\
\hline 1 & $\begin{array}{l}\text { MUY EN } \\
\text { DESACUERDO }\end{array}$ & 0 & 0.00 & \\
\hline \multicolumn{2}{|c|}{ TOTAL } & 40 & 100.00 & \\
\hline
\end{tabular}


Pregunta No. 6 ¿Apoyaría la capacitación continua de los docentes en el área tecnológica?

\section{CUADRO No. 22 REPRESENTANTES LEGALES}

\begin{tabular}{|c|c|c|c|c|}
\hline $\begin{array}{l}\text { ESCAL } \\
\text { A }\end{array}$ & ALTERNATIVAS & $\begin{array}{l}\text { FRECUENCI } \\
\text { A }\end{array}$ & $\%$ & \multirow{7}{*}{$\begin{array}{l}\text { El } 80 \% \text { respondió estar muy de acuerdo a } \\
\text { la interrogante; el } 15 \% \text { de acuerdo; } \\
\text { mientras el } 5 \% \text { opinó que son indiferentes. } \\
\text { Las respuestas indican que es imperante } \\
\text { que los docentes utilicen las tecnologías } \\
\text { educativas para que los estudiantes se } \\
\text { sientan más involucrados dentro del } \\
\text { proceso de aprendizaje y a la vez los } \\
\text { docentes estén en constante actualización y } \\
\text { capacitación cognitiva y pedagógica. }\end{array}$} \\
\hline 5 & $\begin{array}{l}\text { MUY DE } \\
\text { ACUERDO }\end{array}$ & 32 & 80.00 & \\
\hline 4 & DE ACUERDO & 6 & 15.00 & \\
\hline 3 & INDIFERENTE & 2 & 5.00 & \\
\hline 2 & EN DESACUERDO & 0 & 0.00 & \\
\hline 1 & $\begin{array}{l}\text { MUY EN } \\
\text { DESACUERDO }\end{array}$ & 0 & 0.00 & \\
\hline TOTAL & & 40 & 100.00 & \\
\hline
\end{tabular}

Pregunta No. 7. ¿Considera oportuna la acción de las autoridades del plantel de incrementar el uso de laboratorio de Informática a favor de sus representados?

\section{CUADRO No. 23 REPRESENTANTES LEGALES}

\begin{tabular}{|c|c|c|c|c|}
\hline $\begin{array}{l}\text { ESC } \\
\text { ALA } \\
\end{array}$ & ALTERNATIVAS & $\begin{array}{l}\text { FRECUENCI } \\
\text { A }\end{array}$ & $\%$ & \multirow{7}{*}{$\begin{array}{l}\text { El } 72.50 \% \text { respondió en estar muy de } \\
\text { acuerdo; el } 17.50 \% \text { que están de acuerdo } \\
\text { mientras que el } 5 \% \text { respondió estar en } \\
\text { desacuerdo, es decir que un alto } \\
\text { porcentaje de los padres de familia, } \\
\text { apoyan la iniciativa de las autoridades y } \\
\text { docentes de ampliar los laboratorio de } \\
\text { computación con la finalidad de que } \\
\text { exista una mejor preparación tecnológica } \\
\text { de los estudiantes. }\end{array}$} \\
\hline 5 & $\begin{array}{l}\text { MUY DE } \\
\text { ACUERDO }\end{array}$ & 29 & 72.50 & \\
\hline 4 & DE ACUERDO & 7 & 17.50 & \\
\hline 3 & INDIFERENTE & 2 & 5.00 & \\
\hline 2 & EN DESACUERDO & 2 & 5.00 & \\
\hline 1 & $\begin{array}{l}\text { MUY EN } \\
\text { DESACUERDO }\end{array}$ & 0 & 0.00 & \\
\hline$\overline{\text { TOT } A}$ & & 40 & 100.00 & \\
\hline
\end{tabular}

Pregunta No. 8 ¿Colabora con sus representados en apoyarlos con las tareas de carácter tecnológico que realiza en su hogar?

CUADRO No. 24 REPRESENTANTES LEGALES

\begin{tabular}{|l|l|l|l|l|}
\hline $\begin{array}{l}\text { ESCA } \\
\text { LA }\end{array}$ & ALTERNATIVAS & $\begin{array}{l}\text { FRECUENCI } \\
\text { A }\end{array}$ & $\%$ & $\begin{array}{l}\text { El 80\% de los encuestados respondió } \\
\text { estar muy de acuerdo; el 10\% opino } \\
\text { estar de acuerdo; un 5\% se mantuvo } \\
\text { indiferente mientras que el 5\% restante } \\
\text { consideró estar en desacuerdo. Los } \\
\text { padres de familia evidenciaron que } \\
\text { siempre colaboran en las actividades } \\
\text { de sus hijos, inclusive para que } \\
\text { realicen sus tareas en los cyber, al } \\
\text { igual que las investigaciones en } \\
\text { Internet. }\end{array}$ \\
\hline 3 & DE ACUERDO & 42 & 80.00 & 10.00 \\
\hline 1 & INDIFERENTE & 2 & 5.00 & 5.00 \\
\hline
\end{tabular}


Pregunta No. 1 ¿Los docentes de su plantel, están desactualizados en el correcto uso de las Tics?

CUADRO NO. 25 ESTUDIANTES

\begin{tabular}{|l|l|l|l|l|}
\hline $\begin{array}{l}\text { ESC } \\
\text { ALA }\end{array}$ & ALTERNATIVAS & $\begin{array}{l}\text { FRECUENCI } \\
\text { A }\end{array}$ & $\%$ & $\begin{array}{l}\text { Los estudiantes encuestados en un 80\% } \\
\text { respondieron estar muy de acuerdo; el 5\% } \\
\text { estuvo de acuerdo; el 7.50\% se mostró } \\
\text { indiferente ante la pregunta; el 5\% } \\
\text { considero estar en desacuerdo mientras } \\
\text { que el 2.50\% opino estar muy en } \\
\text { desacuerdo. Los resultados revelan que } \\
\text { los docentes de su curso no están } \\
\text { preparados para el uso de programas, } \\
\text { herramientas y aplicaciones informáticas } \\
\text { dentro de su trabajo. }\end{array}$ \\
\hline 3 & DE ACUERDO & 32 & 80.00 & 5.00 \\
\hline 1 & INDIFERENTE & 3 & 7.50 & 5.00 \\
\hline
\end{tabular}

Pregunta No. 2 ¿Es vital que los docentes del octavo grado, se actualicen en el uso de las tecnologías educativas?

CUADRO NO. 26 ESTUDIANTES

\begin{tabular}{|c|c|c|c|c|}
\hline $\begin{array}{l}\text { ESCA } \\
\text { LA }\end{array}$ & ALTERNATIVAS & $\begin{array}{l}\text { FRECUENCI } \\
\text { A }\end{array}$ & $\%$ & \multirow{7}{*}{$\begin{array}{l}\text { El } 75 \% \text { de los estudiantes encuestados } \\
\text { respondió estar muy de acuerdo; el 5\% } \\
\text { contestó estar de acuerdo; el } 7.50 \% \text { están } \\
\text { indiferentes; el } 5 \% \text { en desacuerdo mientras } \\
\text { que el restante } 7.50 \% \text { están muy en } \\
\text { desacuerdo. Se deduce que la mayoría } \\
\text { considera que es imperante que los } \\
\text { docentes de octavo grado se actualicen en } \\
\text { el uso de las tecnologías educativas por } \\
\text { cuanto elevaría la calidad de la educación } \\
\text { en el colegio Aguirre Abad. }\end{array}$} \\
\hline 5 & $\begin{array}{l}\text { MUY DE } \\
\text { ACUERDO }\end{array}$ & 30 & 75.00 & \\
\hline 4 & DE ACUERDO & 2 & 5.00 & \\
\hline 3 & INDIFERENTE & 3 & 7.50 & \\
\hline 2 & EN DESACUERDO & 2 & 5.00 & \\
\hline 1 & $\begin{array}{l}\text { MUY EN } \\
\text { DESACUERDO }\end{array}$ & 3 & 7.50 & \\
\hline TC & & 40 & 100.00 & \\
\hline
\end{tabular}

Pregunta No. 3 ¿Actualmente las clases que reciben son dinámicas, agradables y utilizan tecnología apropiada?

CUADRO NO. 27 ESTUDIANTES

\begin{tabular}{|l|l|l|l|l|}
\hline $\begin{array}{l}\text { ESC } \\
\text { ALA }\end{array}$ & ALTERNATIVAS & FRECUENCIA & $\%$ & $\begin{array}{l}\text { El 60\% de los estudiantes respondieron } \\
\text { muy de acuerdo; el 17.50\% están de } \\
\text { acuerdo; el 5\% son indiferentes; un }\end{array}$ \\
\cline { 1 - 4 } & $\begin{array}{l}\text { MUY DE } \\
\text { ACUERDO }\end{array}$ & 24 & $60.50 \%$ en desacuerdo mientras que el \\
restante 5\% muy en desacuerdo. Los \\
resultados indican que existen criterios \\
divididos sobre la forma de recibir las \\
clases, dado que muchos docentes siguen \\
con métodos tradicionalistas.
\end{tabular}


Pregunta No. 4 ¿Apoyaría la realización de talleres integradores para conocer el contenido de una guía sobre el correcto uso de las Tics, para aplicarla en el octavo grado del colegio?

\section{CUADRO NO. 28 ESTUDIANTES}

\begin{tabular}{|c|c|c|c|c|}
\hline $\begin{array}{l}\text { ESC } \\
\text { ALA }\end{array}$ & ALTERNATIVAS & FRECUENCIA & $\%$ & \multirow{7}{*}{$\begin{array}{l}\text { El } 85 \% \text { de los aprendientes, están muy de } \\
\text { acuerdo; el } 10 \% \text { de acuerdo; el } 2.50 \% \\
\text { indiferentes y el restante } 2.50 \% \text { en } \\
\text { desacuerdo con la pregunta planteada. El } \\
\text { mayor porcentaje indica que es importante } \\
\text { se realicen talleres para conocer y difundir } \\
\text { una guía sobre el uso de las Tics a favor de } \\
\text { sus aprendizajes y conocimientos en las } \\
\text { diferentes asignaturas del octavo grado. }\end{array}$} \\
\hline 5 & $\begin{array}{l}\text { MUY DE } \\
\text { ACUERDO }\end{array}$ & 34 & 85.00 & \\
\hline 4 & DE ACUERDO & 4 & 10.00 & \\
\hline 3 & INDIFERENTE & 1 & 2.50 & \\
\hline 2 & EN DESACUERDO & 1 & 2.50 & \\
\hline 1 & $\begin{array}{l}\text { MUY EN } \\
\text { DESACUERDO }\end{array}$ & 0 & 0.00 & \\
\hline \multicolumn{2}{|c|}{ TOTAL } & 40 & 100.00 & \\
\hline
\end{tabular}

Pregunta No. 5 ¿Incrementaría su rendimiento escolar, en el momento que los docentes utilicen las tecnologías educativas?

\section{CUADRO NO. 29 ESTUDIANTES}

\begin{tabular}{|c|c|c|c|c|}
\hline$\overline{\text { ESCALA }}$ & ALTERNATIVAS & FRECUENCIA & $\%$ & \multirow{7}{*}{$\begin{array}{l}\text { El } 82.50 \% \text { de los estudiantes están muy } \\
\text { de acuerdo; el } 12.50 \% \text { de acuerdo; el } \\
2.50 \text { indiferentes; y el restante } 2.50 \% \\
\text { en desacuerdo. Los resultados indican } \\
\text { que los estudiantes se muestran } \\
\text { interesados en incrementar su } \\
\text { rendimiento escolar, en el momento } \\
\text { que los docentes cambien sus métodos } \\
\text { tradicionales y se apoyen en las } \\
\text { herramientas tecnológicas actualizadas. }\end{array}$} \\
\hline 5 & $\begin{array}{l}\text { MUY DE } \\
\text { ACUERDO }\end{array}$ & 33 & 82.50 & \\
\hline 4 & DE ACUERDO & 5 & 12.50 & \\
\hline 3 & INDIFERENTE & 1 & 2.50 & \\
\hline 2 & $\begin{array}{l}\text { EN } \\
\text { DESACUERDO }\end{array}$ & 1 & 2.50 & \\
\hline 1 & $\begin{array}{l}\text { MUY EN } \\
\text { DESACUERDO }\end{array}$ & 0 & 0.00 & \\
\hline TOTAL & & 40 & 100.00 & \\
\hline
\end{tabular}

Pregunta No. 6. ¿Los docentes del octavo grado, utilizan herramientas de Word, Excel, power point, internet para motivar adecuadamente los aprendizajes?

\section{CUADRO NO. 30 ESTUDIANTES}

\begin{tabular}{|c|c|c|c|c|}
\hline $\begin{array}{l}\text { ESCAL } \\
\text { A }\end{array}$ & ALTERNATIVAS & $\begin{array}{l}\text { FRECUENC } \\
\text { IA }\end{array}$ & $\%$ & \multirow{7}{*}{$\begin{array}{l}\text { El } 80 \% \text { de los estudiantes escogieron la } \\
\text { opción muy de acuerdo con la pregunta } \\
\text { planteada; el } 17.50 \% \text { de acuerdo; } \\
\text { mientras que el restante } 2.50 \% \text { son } \\
\text { indiferentes por lo que los docentes } \\
\text { deberían potencializar sus clases a } \\
\text { través de la aplicación de los recursos } \\
\text { Web lo cual crearía una interacción } \\
\text { virtual con sus estudiantes. }\end{array}$} \\
\hline 5 & MUY DE ACUERDO & 32 & 80.00 & \\
\hline 4 & DE ACUERDO & 7 & 17.50 & \\
\hline 3 & INDIFERENTE & 1 & 2.50 & \\
\hline 2 & EN DESACUERDO & 0 & 0.00 & \\
\hline 1 & $\begin{array}{l}\text { MUY EN } \\
\text { DESACUERDO }\end{array}$ & 0 & 0.00 & \\
\hline TOTAL & & 40 & 100.00 & \\
\hline
\end{tabular}


Pregunta No. 7 ¿El uso de material audiovisual ayudaría a comprender adecuadamente las clases de los docentes del octavo grado?

\section{CUADRO NO. 31 ESTUDIANTES}

\begin{tabular}{|c|c|c|c|c|}
\hline $\begin{array}{l}\text { ESCAL } \\
\text { A }\end{array}$ & ILTERNATIVAS & $\begin{array}{l}\text { FRECUENCI } \\
\text { A }\end{array}$ & $\%$ & \multirow{7}{*}{$\begin{array}{l}\text { El } 72.50 \% \text { de los estudiantes } \\
\text { respondieron muy de acuerdo; el } 12.50 \% \\
\text { de acuerdo; el } 5 \% \text { indiferentes, el restante } \\
10 \% \text { en desacuerdo. Analizados los } \\
\text { resultados es necesario que los docentes } \\
\text { utilicen en sus clases recursos } \\
\text { audiovisuales para la explicación de un } \\
\text { tema de la asignatura que conste en la } \\
\text { malla curricular. }\end{array}$} \\
\hline 5 & MUY DE ACUERDO & 29 & 72.50 & \\
\hline 4 & $\overline{\text { DE ACUERDO }}$ & 5 & 12.50 & \\
\hline 3 & INDIFERENTE & 2 & 5.00 & \\
\hline 2 & EN DESACUERDO & 4 & 10.00 & \\
\hline 1 & $\begin{array}{l}\text { MUY EN } \\
\text { DESACUERDO }\end{array}$ & 0 & 0.00 & \\
\hline U1AL & & 40 & 100.00 & \\
\hline
\end{tabular}

Pregunta No. 8 ¿El docente debería utilizar las aplicaciones de un computador y redes sociales para incrementar los aprendizajes de los estudiantes?

\section{CUADRO NO. 32 ESTUDIANTES}

\begin{tabular}{|c|c|c|c|c|}
\hline $\begin{array}{l}\text { ESCAL } \\
\text { A }\end{array}$ & ALTERNATIVAS & $\begin{array}{l}\text { FRECUENCI } \\
\text { A }\end{array}$ & $\%$ & \multirow{7}{*}{$\begin{array}{l}\text { Un } 72.50 \% \text { de los estudiantes } \\
\text { contestaron muy de acuerdo; el } 12.50 \% \\
\text { de acuerdo; un } 5 \% \text { indiferentes y el } \\
\text { restante } 10 \% \text { en desacuerdo. Las } \\
\text { diferentes respuestas, indican que es } \\
\text { indispensable que los docentes utilicen } \\
\text { un computador con sus aplicaciones y } \\
\text { que estén inmersos en las redes sociales } \\
\text { para mejorar las motivaciones } \\
\text { académicas en las diferentes asignaturas. }\end{array}$} \\
\hline 5 & MUY DE ACUERDO & 29 & 72.50 & \\
\hline 4 & DE ACUERDO & 5 & 12.50 & \\
\hline 3 & INDIFERENTE & 2 & 5.00 & \\
\hline 2 & EN DESACUERDO & 4 & 10.00 & \\
\hline 1 & $\begin{array}{l}\text { MUY EN } \\
\text { DESACUERDO }\end{array}$ & 0 & 0.00 & \\
\hline TOTAL & & 40 & 100.00 & \\
\hline
\end{tabular}




\section{Discusión}

Desde el inicio de la investigación, el maestrante planteó el supuesto a conseguir, para ello determinó la Hipótesis, la misma que al término del capítulo IV se concluyó de acuerdo a la aplicación de diferentes técnicas de recolección de datos que la misma es positiva y verdadera, ya que, los involucrados expresaron mayoritariamente que una guía cognitiva sobre las Tics, permitiría mejorar substancialmente los aprendizajes en las diferentes asignaturas de la malla curricular de la unidad educativa fiscal Aguirre Abad y de manera particular de los aprendientes del octavo grado.

Efectivamente, mediante la observación empírica, las conversaciones con los diferentes estamentos de la comunidad educativa y la aplicación de las encuestas a las autoridades, docentes, representantes legales y estudiantes, permitieron recoger información idónea en relación al problema pedagógico y a la propuesta diseñada por el autor de la tesis, se aplicó la Estadística Descriptiva y los resultados se plasmaron en los cuadros, gráficos y los análisis porcentuales y pedagógicos que terminaron demostrando la validez del informe final redactado por el maestrante y que a la postre sería de mucha utilidad práctica para el adelanto académico de las institución educativa.

Se demostró que ampliando el radio de acción de vinculación de las tecnologías educativas a los docentes del colegio, los estudiantes estarían dispuestos a prepararse mejor y coordinar entre ellos las diferentes tareas de investigación por medio de Internet y desarrollar mejores competencias cognitivas que fortalecerían la formación integral de los aprendientes del plantel.

\section{La propuesta}

Título: Desarrollar estrategias para la aplicación de una guía práctica relacionada al uso correcto de las tecnologías de información y comunicación, buscando potenciar la formación integral de los estudiantes.

Objetivo: Incorporar en la institución educativa, una guía cognitiva sobre el correcto uso de las tecnologías de información y comunicación y sus utilitarios, mediante la utilización de recursos tecnológicos en el aula para mejorar el desempeño de los estudiantes 
Beneficiarios de la propuesta: autoridades, hodogogos, representantes legales, aprendientes, comunidad, descripción de la propuesta

Diseño: La propuesta está diseñada de la siguiente manera:

\section{BLOQUE 1}

Estrategia: Sopa de Letras

Tema: Documentos en Word

\section{BLOQUE 2}

Estrategia: Brainstorming (Lluvia de ideas)

Tema: Presentaciones en PowerPoint y Creaciones en Publisher

\section{BLOQUE 3}

Estrategia: Resolución de Problemas

Tema: Hoja de cálculo en Excel

\section{BLOQUE 4}

Estrategia: Rol play

Tema: Blog

\section{BLOQUE 5}

Estrategia: Foro

Tema: Internet y Redes sociales 


\section{SEGUIMIENTO DE LA PROPUESTA}

El maestrante, con la finalidad de comprobar las acciones a seguir para el fiel cumplimiento de los contenidos de la propuesta, considera pertinente efectuar lo siguiente:

Evaluar el desarrollo del contenido de la propuesta que realicen en forma separada los docentes, autoridades, representantes legales y estudiantes.

Integración de equipos humanos interdisciplinarios

Detallar los contenidos de los cursos de computación de forma constante para estar al día con las nuevas herramientas y tecnológicas.

\section{Conclusiones.}

Se comprobó la veracidad y planteamiento de la Hipótesis en la presente investigación cualitativa, dado que la comunidad educativa, apoyó la propuesta diseñada por el autor de la tesis de maestría.

El objetivo general y los objetivos específicos de la investigación se cumplieron a cabalidad, demostrándose la claridad de lo, planeado en la estructura de la tesis, utilizando para ellos la metodología adecuada, alcanzando logros en el mejoramiento de las relaciones docenteaprendiente.

Las autoridades de la unidad educativa fiscal Aguirre Abad de la ciudad de Guayaquil, deben incluir en sus planificaciones anuales la capacitación pedagógica del personal docente de la institución, buscando robustecer criterios idóneos vinculados a las nuevas teorías pedagógicos, de manera muy especial los conocimientos básicos sobre el constructivismo en materia de aprendizajes significativos. 
Los Hodogogos, deben constituirse en modelos ejemplares a seguir por los estudiantes, buscando se apliquen los conceptos relacionados a la horizontalidad del aprendizaje

Los representantes legales, asumirán su rol protagónico que la sociedad ecuatoriana así lo impone. Los hábitos de comportamiento y de estudio, así como las normas de sana convivencia en el hogar deben ser aplicados para fortalecer los conceptos básicos vinculados a los valores morales, éticos y humanos, en beneficio de sus representados.

Los docentes están obligados a desarrollar las actividades enmarcados en el uso adecuado de las tecnologías que están presentes en nuestro medio y que tienen impacto en la vida cotidiana. las Tecnologías de la Información y Comunicación como instrumentos transmisores o procesadores de informaciones deben ser aplicadas por los estudiantes para estar al día con el mundo tecnológico. Los Hodogogos, deben dominar las estrategias activas dentro del proceso de aprendizaje que incluyan la tecnología educativa

\section{Bibliografía.}

Carnoy, M. (2004). Las TIC en la enseñanza: posibilidades y retos. UOC. La universidad virtual.

Castillo, S. (2008). Propuesta pedagógica basada en el constructivismo para el uso óptimo de las TIC en la enseñanza y el aprendizaje de la matemática. Revista Latinoamericana de investigación en matemática educativa.

Coll, C. (2007). TIC y prácticas educativas: realidades y expectativas. Ponencia presentada en el XXIII Congreso Semana Monográfica de Educación.

Collins, A. (1998). El potencial de las tecnologías de la información para la educación. En C. Vizcarro \& J. León (Coords.), Nuevas Tecnologías para el aprendizaje (pp. 29-46). Madrid: Pirámide.

De Pablos Pons, J. \& Jiménez Cortés, R. (2007). Buenas prácticas con TIC apoyadas en las Políticas Educativas: claves conceptuales y derivaciones para la formación en competencias ECTS.

Escontrela Mao, R. \& Stojanovic, L. (2004). La integración de las TIC en la educación: Apuntes para un modelo pedagógico pertinente. Revista de pedagogía, 11, (02), 171-194.

Fandos, M., Jiménez, J. \& González, P. (2003). Estrategias didácticas en el uso de las Tecnologías de la Información y la Comunicación. Revista Acción Pedagógica, 11, (1). 
Eduardo G. Guadalupe-Coronel; Jonathan H. Guadalupe-Beltrán; Eduardo S. Guadalupe-Beltrán

Màrques, P. (2000). Impacto de las TIC en la educación. Tecnología educativa - Web Pere Marquès.

Ministerio de Educación del Ecuador (2011). Estándares TIC del estudiante de Bachillerato. Documento en preparación. Quito: Ministerio de Educación del Ecuador. 